\title{
AMCP Partnership Forum: Managing Total Cost of Care Through Medical and Pharmacy Data Integration
}

\section{SUMMARY}

Value-driven health care models that aim to improve patient outcomes while managing total costs of care require better access to integrated health care data. Health care organizations that integrate data from multiple platforms (e.g., pharmacy, medical, electronic medical records, and lab data) can better streamline and guide effective, efficient, and coordinated utilization and care management programs.

To address strategies for integrating health care data, the Academy of Managed Care Pharmacy convened a forum in Baltimore, MD, on September 25-26, 2018. The goal of the forum was to develop a roadmap for managed care entities interested in (a) managing medications across the spectrum of health care-regardless of whether the medications are covered by a medical or pharmacy benefit; (b) driving performance improvements in clinical quality with integrated datasets; (c) streamlining care transitions and case management; and (d) facilitating the development of datasets formatted for use in value-based care contracts.

Forum deliberations highlighted the need for data integration in valuebased health care systems, explored making data actionable through rapid and predictive analytics of integrated datasets, identified the challenges of doing so, and developed a practical roadmap for managing the total cost of care through data integration.

Health care leaders representing health plans, integrated delivery systems, pharmacy benefit managers, employers, the federal government, technology companies, data integrators, standards development leaders, and biopharmaceutical companies participated in the forum, which was supported in collaboration with Abbvie, Alkermes, Amgen, Bayer, Boehringer Ingelheim, Genentech, Merck, National Pharmaceutical Council, PhRMA, Takeda, and Xcenda. These proceedings represent common themes and comments from individual participants; they are not necessarily endorsed by all attendees, nor should they be construed as reflecting group consensus.

J Manag Care Spec Pharm. 2019;25(3):292-99

Copyright $\odot 2019$, Academy of Managed Care Pharmacy. All rights reserved.

I ntegrating health care data to inform and improve care can provide benefits to many stakeholders. For example, realLtime access to clinical and cost information could allow patients to be better informed about their care and support individual decision making. Providers could have the ability to assess clinical outcomes, practice efficiency and utilization patterns, evaluate patient care and costs holistically, and engage in effective team-based care. Employer groups and benefit consultants could have additional information to inform marketplace comparisons. Managed care organizations and integrated care delivery networks could partner to use integrated data to manage total costs of care, better manage disease states and patient care, inform the design of benefit packages, implement shared risk programs and value-based contracts, and guide population management interventions.
Data integration is crucial to actualizing health care models that reward value over volume. Payment models that are based on patient outcomes and the total cost of care must have the ability to analyze data from pharmacy and medical benefits, as well as other sources. To address these issues, the Academy of Managed Care Pharmacy (AMCP) convened a stakeholder forum, "Managing Total Cost of Care Through Medical and Pharmacy Data Integration" in Baltimore, MD, on September 25-26, 2018. Forum participants emphasized that data can be used to achieve all aspects of the triple aim-improving patient care (including quality and satisfaction), improving the health of populations, and reducing the cost of health care.

Participants reviewed information regarding the role of data integration in addressing total cost of care. Acknowledging that the top 5\% of costliest patients account for about half of expenditures, many strategies that are aimed at managing the total cost of care often focus on these patients. ${ }^{1}$ Additionally, it is important to implement preventive strategies to optimally manage patients with chronic conditions to reduce their future risk of becoming high-cost patients in the future. Strategies to provide more efficient care focus on reducing preventable emergency department visits and hospitalizations; inappropriate specialty referrals, medication use, or lab testing; unwarranted diagnostic or surgical procedures; and inappropriate site-of-service usage (e.g., performing an infusion at a hospital instead of a free-standing radiology center). The ability to integrate, access, and evaluate medical and pharmacy data from a variety of sources facilitates the implementation of interventions that support these strategies.

Forum participants envisioned a future in which data will be used to guide clinically effective solutions and population management interventions and serve as a tool for shared risk programs. Access to data and related insights would be seamless and information most useful to users readily accessible at the point of service to provide real-time data-based decision making across care teams. Successful data integration will also support more streamlined, efficient, and effective longitudinal care. Patients will be able to access information about clinical options and the costs of those options.

\section{A Roadmap for Data Integration}

$\overline{\text { Forum participants were asked to outline steps that managed }}$ care organizations can take to integrate medical and pharmacy data to achieve the desired vision for the future. Participants developed a roadmap to guide entities through recommended actions to promote data integration, address the challenges, and work toward actionable data needed to manage the total cost of care. 


\section{TABLE 1 Leadership and Governance Roadmap Goals and Challenges}

Strategies to Achieve Goals

Identify the problem and describe

why the organization needs to

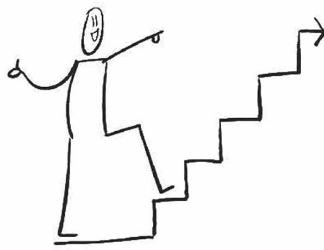

- Establish organizational priorities, policies, and procedures that ensure organization-wide commitment and buy-in to addressing the total cost of care.

- Department structures and employee roles are supported by clear expectations and resource commitments to enable the goal of addressing the total cost of care.

\begin{tabular}{|c|} 
address it \\
$\begin{array}{c}\text { C-suite commits to pursue a strategic } \\
\text { plan that is focused on value and } \\
\text { defines expected outcomes }\end{array}$ \\
\hline
\end{tabular}

Create a cross-functional team, including its structure, leadership, and approach to data governance

and approach to data governance

Communicate priorities and align incentives with internal and external stakeholders

Assess and report performance

Key Tactics to Achieve Goals

- Create a business case

- Identify risks and opportunities

- Prioritize short-term and long-term goals

- Set a vision

- Confirm the financial commitment

- Identify the short-term and long-term expected outcomes

- Identify the steering committee membership and accountability

- Define roles and responsibilities

- Identify staffing needs and champions and empower them to do the work

- Identify internal and external stakeholders

- Seek input and provide education

- Identify and address barriers to incentives

- Align short-term and long-term goals

- Define key performance indicator metrics

- Set realistic time frames

- Report to internal and external stakeholders

\section{Challenges}

\section{All MCOs:}

- Time lag for data access, particularly for medical data

- External environment of mergers and acquisitions

- Benefit carve-outs
- Internal and external capabilities for data consumption or management

- Misaligned incentives

- Resources, personnel limitations or skills/qualifications, partners

- Data rights

\section{Strategies to Address Challenges}

- "Yelp" for health care

- More transparency for cost of care information for all stakeholders, including patients

- Have discussions with vendors early

- Creating key partnerships where needed

- Change management best practices

MCO = managed care organization

As part of the roadmap, participants addressed 4 functional areas and associated goals for the roadmap: leadership and governance, finance and business, health information technology (IT), and care delivery (benefits and coordination). Participant recommendations for goals, strategies, and tactics to achieve goals within each of these 4 functional areas, as well as strategies to address potential challenges, are presented in Tables 1-4.

Participants grappled with the order of functional areas within the roadmap. The roadmap was initially conceived to begin with leadership and governance, then progress to care delivery, health IT, and finally finance and business (Figure 1). However, participants stressed that the advancement of data integration will require strategic planning and operational work to be successful. Upon further discussion, they determined that the steps for advancing data interoperability should follow a different order:

1. Leadership is needed to drive initiatives forward, establish expectations, and promote culture that is supportive of data integration, as well as to assign responsibility for various activities and hold individuals accountable for completing these tasks.

2. Assessing the financial effect of an organizational approach to managing total cost and developing a business case to support investments would be the next step. This would be driven by leadership and provide critical feedback and resources to guide next steps.

3. Development and implementation of technology solutions to allow for integration would be the next step, which would then allow for step 4.

4. Data integration to be a component of care delivery that allows clinicians to assess patients efficiently and comprehensively to manage total costs.

Finally, participants indicated that these 4 components form a cycle rather than a linear step-wise approach.

Several themes emerged for goals and strategies that are important for achieving these functional goal areas, including definitions and standards, planning for effective use of analytics, and standardization of datasets.

\section{Definitions and Standards}

Participants acknowledged several needs related to developing definitions and standards, including the need to identify priority data types and develop definitions that are mutually agreed upon by stakeholders, and then formatting the data in a manner that is actionable for decision making. Which costs and cost offsets should be included and the time frame for 
TABLE 2 Business and Finance Roadmap Goals, Tactics, and Challenges

Strategies to Achieve Goals

Leadership buy-in and socializing the message internally for consistency

- Ensure that goals for financial stewardship and profit margins take into consideration the impact to total cost of care.

- That strategies, initiatives, and business practices are aligned across the organization, clients and/or other relevant entities to encourage the evaluation of how decisions for benefit design of product offerings impact total costs for patient care as a whole.

Invest technology platforms and human resources to support total cost of care integration

care approach and collaboration contracts

Develop timelines and metrics for ROI for the business case
Key Tactics to Achieve Goals

- Develop a business case through competitive assessment of the market

- Develop a purpose message for internal messaging to explain why this is important to stakeholders

- Listen to our clients and their needs regarding total cost of care

- Assess currently available tools, data, and resources

- Identify needs for tools, data, and human resources and perform gap assessments and develop job descriptions

- Redirect the budget based on identified resource needs

Align financial incentives with $\quad$ - Determine key stakeholders early in the process and assess how they will external vendors to total cost of manage potential change

- Conduct pilots to test new programs

- Negotiate new contracts as needed

- Outline expected timeline for ROI

- Develop different metrics for different stakeholders

- Use an iterative process

\section{Challenges}

\section{Strategies to Address Challenges}

\section{All MCOs:}

- Competing priorities within an organization and within a group of organizations

- Technology platform differences across organizations

- Limited budgets and silos

Third-party entities

(PBM, MTM provider):

- Lack of data that requires need to purchase specific datasets (e.g., lab)

\section{Health plans:}

- Need to manage multiple client needs and expectations and age demographics_-not all may desire TCC

- Multiple LOBs with different needs and regulations

Other managed care settings:

- High investment HIT program with longer-term outcomes

- Overstandardization of documentation in EHRs diminishes the value of the unique narrative data about the specific patient for attending clinicians
- Government programs, ACO, Medicaid programs

- Risk pools to mitigate the unknown

- Bring key stakeholders to the table

- Align incentives to outcomes

- Keep the patient at the center

$A C O=$ accountable care organization; EHR = electronic health record; HIT = health information technology; $L O B=$ lines of business; $M C O=$ managed care organization; $M T M=$ medication therapy management; $P B M=$ pharmacy benefit manager; $R O I=$ return on investment; $T C C=$ total cost of care.

analyzing such costs, were also identified as important issues to consider when defining total cost of care for an organization.

Participants recognized that different stakeholders can have different economic perspectives; for example, whether to include "indirect" costs incurred by health plans or pharmacy benefit managers (PBM), such as lost employment or disability payments, in total cost calculations must be determined, since these costs have important implications for many stakeholders. The role of rebates for prescription products in overall pricing structures must also be considered, since historically, the use of gross or list price in total cost of care calculations can substantially overestimate actual costs after rebates. Although cost perspective may vary by organization, participants agreed that one definition for total cost should be used across an organization or between partners managing the health of a group of patients.

When defining time frames, it is important to recognize that some interventions are costlier in the short term but provide longterm savings and that stakeholders may operate within different time frames. Thus, the effect of an intervention on total cost will vary depending on the time frame being considered. Participants observed that actuaries generally assess costs on a quarterly or 1- to 2-year basis, whereas the value of many preventive interventions are achieved over several years or decades.

The value of an intervention in terms of patient outcomes must also be considered. Participants noted complexities associated with assessing patient outcomes and related aspects of quality.

\section{Planning for Effective Use of Analytics}

Participants felt that predictive analytics combined with clinical expertise is helpful for effectively using data. Participants recommended collaborations among clinicians, data scientists, and IT programmers to develop such analytic tools that will allow for effective use of data. Participants observed that any data analysis should be purposeful and aligned with clearly identified goals to allow leadership to assess return on investment and to evaluate end-user adoption. 


\section{TABLE 3 Health Information Technology Roadmap Goals, Tactics, and Challenges}

\section{Health Information \\ Technology Goals}

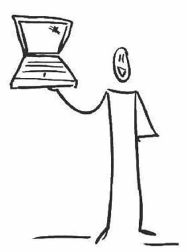

- Use the most efficient technological means to access available data sources necessary to evaluate how organizational processes and/or services impact total cost of care and patient outcomes.

- Establish processes to enable evaluations of both pharmacy and medical benefit information to inform delivery of care.

\begin{tabular}{|} 
Strategies to Achieve Goals \\
$\begin{array}{c}\text { Adopt an established data definition } \\
\text { for total cost of care }\end{array}$ \\
\hline
\end{tabular}

Adopt an established definition for patient outcomes

a

Adopt a common data model to integrate EHR and claims data

Adopt a common data model to incorporate patient-generated data

Build, maintain, and optimize infrastructure to support actionable insights and continuous quality improvement

\section{Key Tactics to Achieve Goals}

- Confirm leadership's needs for measurement to achieve organizational objectives and timelines

- Create a cross-functional team to agree on and adopt definitions for total cost of care and relevant patient outcomes

- Perform a gap analysis of organization-owned or accessible datasets to capture total cost of care and patient outcomes

- Develop a business plan for leadership, outlining steps to close gaps in data, IT infrastructure, and expertise to access all necessary datasets

- Institute proof of concept pilot process with a subset of business partners or patient data to test and confirm ROI and adjust the plan for scaling

- Establish data use agreements and contracts that ensure real-time access to information

- Ensure appropriate controls and safeguards to access information

- Identify expertise to lead a "build or buy" analysis

- Identify and communicate IT resources, analytics, and training needs

- Ensure internal capabilities for secure data warehousing

- Establish interoperable connections to access data at the source to ensure actionable insights

- Confirm leadership needs for measurement to achieve organizational objectives and timelines

- Create a cross-functional team to agree on and adopt definitions for total cost of care and relevant patient outcomes

- Perform a gap analysis of organzation-owned or accessible datasets to capture total cost of care and patient outcomes

- Develop a business plan for leadership, outlining steps to close gaps in data, IT infrastructure, and expertise to access all necessary datasets

- Institute proof of concept pilot process with a subset of business partners or patient data to test and confirm ROI and adjust the plan for scaling

- Establish data use agreements and contracts that ensure real-time access to information

- Ensure appropriate access controls and safeguards to access information

Establish processes to support actionable insights and continuous quality improvement

\begin{tabular}{|c|c|c|}
\hline Challenges & \multicolumn{2}{|c|}{ Strategies to Address Challenges } \\
\hline $\begin{array}{l}\text { - Patient matching } \\
\text { - Data gaps } \\
\text { - Partnership management } \\
\text { - Incorporating unstructured data in EHRs } \\
\text { - Lack of longitudinal data } \\
\text { - Analytics-lack of foundational skills and over- } \\
\text { whelmed by Big Data } \\
\text { - Patient-centricity }\end{array}$ & $\begin{array}{l}\text { - Deterministic matching } \\
\text { - Universal patient ID } \\
\text { - Prioritization of gap analysis } \\
\text { - Be able to consume data in its existing format } \\
\text { - Involvement with standards development } \\
\text { (HL7/FIHR, NCPDP) } \\
\text { - Align incentives }\end{array}$ & $\begin{array}{l}\text { - Share risk } \\
\text { - Shared decision making } \\
\text { - Implement structured data and capture } \\
\text { - Machine learning/natural language processing } \\
\text { - Cohort comparison } \\
\text { - Mobile health devices }\end{array}$ \\
\hline
\end{tabular}

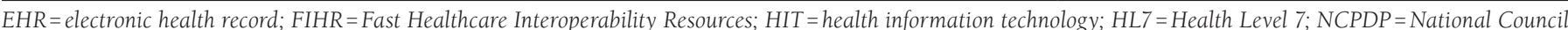
of Prescription Drug Programs; ROI= return on investment.

Some clinicians will need to be trained in data analytics and interpretation so that they will be prepared to interpret the data and apply it to practice. User friendly interfaces, such as scorecards or dashboards, can be used to make data insights more accessible. However, access to data must be balanced with practical utility of data so that front-line clinicians and decision makers are not overwhelmed with more information than they can reasonably process and apply. 


\section{TABLE 4 Care Delivery (Benefits and Care Coordination) Roadmap Goals, Tactics, and Challenges}

Care Coordination Goals

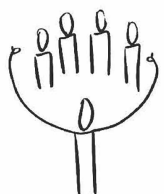

- Optimize individual and population patient outcomes by using all tools and services available to manage patient care

- Access to and provision of benefits and services are evidence-based and cost-effective.
Strategies to Achieve Goals

Key Tactics to Achieve Goals

- Identify the key stakeholders

- Empower a champion with an executive sponsor to remove barriers

- Link the program to organizational priorities and goals

- Create a business plan with resources, resource commitments, and outline the process

- Identify the care teams and roles, gaps, and leader

Integrated care teams that touch all aspects of the patient's care

- Training and coordinating patient-focused communication

- Create visibility of accessible data into care team workflow

- Engage the patient and care team in shared decision making

- Understand their role in decision making

Identify and support the health care provider and caregiver

- Create a line of communication between caregiver and care team

- Educate caregiver

- Define meaningful outcomes, how to measure, and training

- Align the outcomes with the mission, vision, and core values

- Mitigate avoidable consequences through a CQI process

- Understand role in decision making

- Create line of communication to care team

- Educate and provide resources

\section{Challenges}

- Sliced environment, both internally and externally with vendor partners

- Need a leader/champion

- Lack of awareness of importance of clinical data

- No direct access to EHR for some members of the care team

- Hard to find qualified staff

- Absence of a caregiver
- Lack of health literacy

- Multitude of metrics around satisfaction, cost, and quality

- Managing patient/family expectations

- Educating patient/family on health care data

- Cost of network/speciality services is often unknown and lack of understanding of benefits

\section{Strategies to Address Challenges}

- Interdisciplinary teams

- Training to support teams to provide professional development, internal promotion, and ongoing reinforcement

- Design training programs to promote internally

- Create training programs for professional development

- Keep measurement simple

- Shared decision making supported by appropriate data/information

$C Q I=$ continuous quality improvement; $E H R=$ electronic health record; $M C O=$ managed care organization.

\section{Standardization of Datasets}

Some types of data are more structured than others. For example, laboratory data are often structured, whereas notes from a clinician describing subjective impressions of patients are more open-ended. Participants noted that various data elements provide different snapshots of the patient and do not necessarily provide the entire picture. Therefore, it is important to assemble and present relevant datasets in a comprehensible format that will inform clinical and coverage decisions.

Different methods for categorizing data (e.g., different time periods) can result in different interpretations of data. Participants noted that, if patients visit providers and specialists from different networks and obtain prescriptions from multiple sources, the provider networks may have different sets of data, for the same patient. Therefore, sharing of interoperable datasets across all systems can provide a more complete picture.

Health care data standards support efforts to integrate data, and some data transfer standards have already been developed.
For example, the National Council for Prescription Drugs Program (NCPDP) develops national standards for real-time, electronic exchange of health care information. These standards are used widely throughout the pharmacy industry for prescribing in the ambulatory environment and dispensing, monitoring, managing, and paying for medications.

Health Level-7 (HL7) provides a framework and standards for the transfer and integration of clinical and administrative data that are stored in electronic health records (EHRs). Fast Healthcare Interoperability Resources (FHIR) is an HL7 standard and aims to simplify data exchange among health care applications and promote greater interoperability of systems. Many industry leaders are currently working to implement FHIR-based solutions. Additionally, efforts to integrate systems that use NCPDP and FHIR standards are already being considered and would help streamline integration of medical and pharmacy data. 


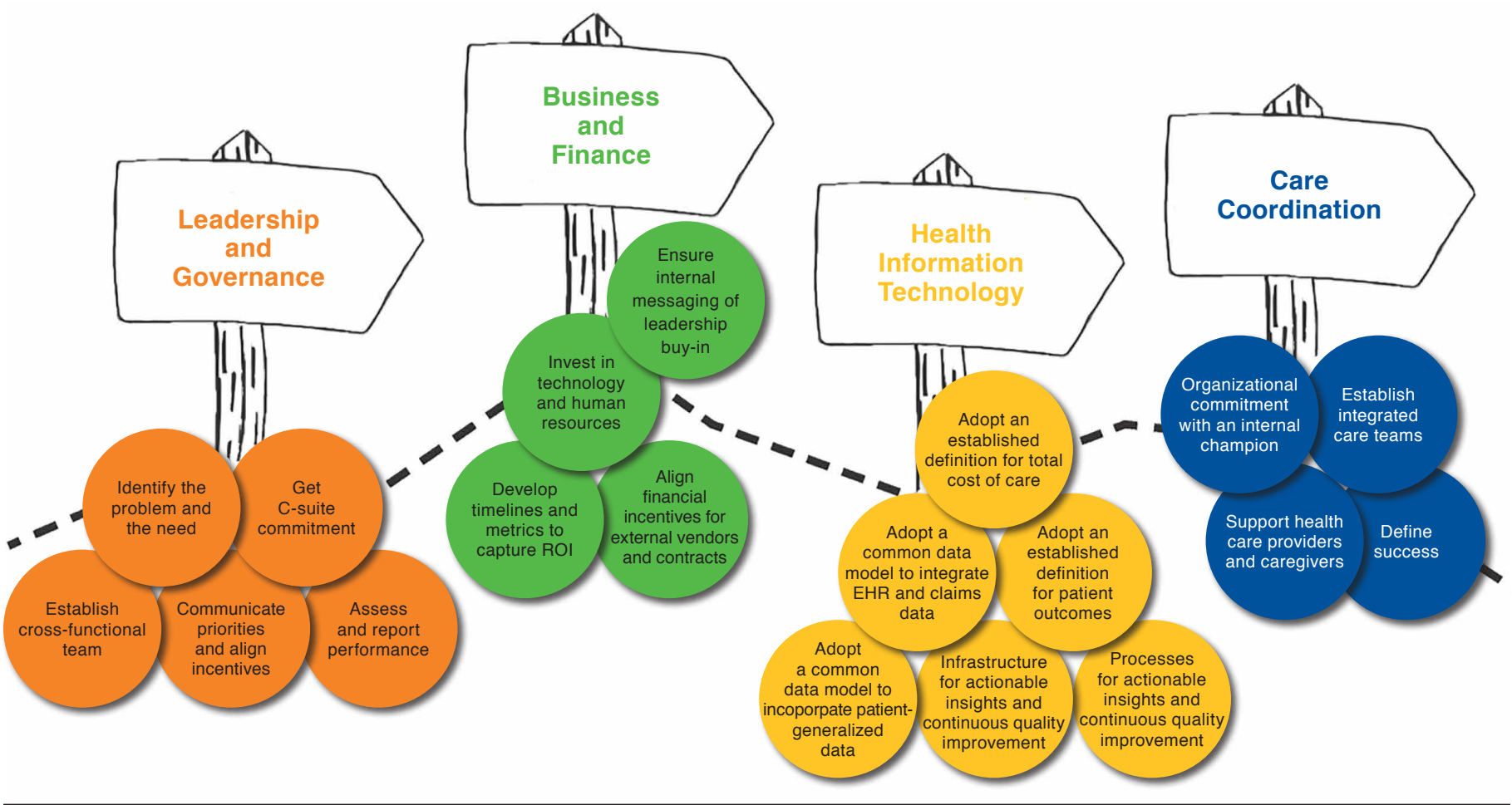

Note: This image was modified from the original artwork, which is available online at: http://www.amcp.org/Sept2018PartnershipForumVisualSummary.pdf. $E H R=$ electronic health record; $R O I=$ return on investment.

Participants noted that private industry initiatives (e.g., the Da Vinci Project) are building on these platforms with the goal of promoting interoperability across value-based care stakeholders and developing interoperable solutions nationally. ${ }^{2}$ Blockchain technology, which allows a growing list of records (blocks) to be linked using cryptography, was also proposed by participants as a potential solution to allow interoperability.

Participants observed that the federal government, particularly the Centers for Medicare \& Medicaid Services (CMS), has been a leader in driving the shift toward value-based purchasing and has several efforts underway that support longitudinal data integration. Innovative models are emerging from CMS and some state Medicaid programs that may help inform the efforts of other entities. Some integrated care delivery networks and accountable care organizations also have integrated pharmacy and medical systems. Participants noted that organizations with developed models already engage in value-based contracts and that there are opportunities to identify best practices and share them with others.

\section{Challenges Facing Data Integration}

In addition to exploring goals, strategies, and tactics for advancing data integration, participants also explored challenges to integrating data from different sources and explored strategies for addressing those challenges. In August 2018, AMCP members (managed care organizations, accountable care organizations, integrated delivery networks, PBMs, employer groups, and others; $\mathrm{N}=67$ ) were surveyed regarding barriers to integrating pharmacy and medical data (unpublished data, Academy of Managed Care Pharmacy, Integrating Pharmacy and Medical Data to Better Manage the Total Cost of Care survey results, August 2018). This survey found that coding differences, lack of access to real-time data, and lack of data standardization were the most commonly cited barriers. Barriers that were identified in the survey, listed in order of most commonly cited to least commonly cited, are as follows:

1. Coding differences between pharmacy and medical data

2. Lack of access to real-time data

3. Lack of sufficient data standardization

4. Costs for implementing data integration technology 
5. Plan sponsor benefit carve outs

6. Lack of access to specific datasets

7. Lack of experience with data integration or data warehouses

8. Privacy and security requirements

9. Lack of a supportive business or regulatory environment

10. Issues with health information exchanges

Participants discussed the barriers presented in the survey and also explored other challenges for consideration in the roadmap.

\section{Sources of Data}

Datasets from different systems typically are stored in different formats, which complicates efforts to analyze and integrate them. Participants observed that even within a single domain (e.g., medical, pharmacy, laboratory, imaging), patients may visit multiple providers that use different EHR systems and that those systems are often siloed. Different EHR systems may have been customized with organization specific formats and templated, complicating efforts to combine data. For example, within capitated care delivery models, data related to encounters may be less detailed than in other delivery models because providers may not be required to submit claims for specific interventions.

EHRs are not necessarily accessible across provider systems (e.g., from one medical system to another). Even within a single organization, there may be data silos. For example, different departments within a single payer may use different IT systems and have challenges sharing data effectively among themselves. Combining data from different external systems poses additional challenges.

Additional sources of health care data, such as laboratory, dental, vision, radiology, chiropractic, and mental health, as well as mobile health (mHealth) devices, such as patient wearable and remote physiologic monitoring devices, are also important to consider when assessing patient care and developing strategies for integrating data. The integration of patient-reported outcomes is also necessary to support the development of patient-centered interventions and to capture subjective improvements that matter to patients, such as pain relief and improvement in activity levels. Care providers working outside of medical offices (e.g., community pharmacists) generally do not have access to EHR systems. Nontraditional care providers (e.g., acupuncturists, herbalists) are increasingly involved in patient care and also are not generally integrated with clinical and claims systems used by other physical or behavioral providers.

Participants stressed that social determinants of health have an important influence on patient care and that community services data could inform decision making. For example, area aging agencies or groups that provide services to the homeless or underserved populations may have important information about patients that could also be integrated to better manage care coordination, care delivery, and the total cost of care for patients.
Participants identified ownership and access rights for data as another issue that will need to be addressed when accessing, combining, or integrating data from multiple sources. Health Insurance Portability and Accountability Act (HIPAA) requirements for patient privacy must also be considered when determining whether the data that are shared must be deidentified and aggregated.

\section{The Business Case for Integration}

Data integration requires substantial investments to build the necessary data infrastructure, and organizations need to be able to justify the expenditures. Entities that are at financial risk for quality and value likely have the greatest incentives to pursue data integration investments, whereas those that are operating under traditional fee-for-service contracts may not have as many incentives to manage total cost of care or integrate data across departments or sites of care.

Drivers that support the investment of resources include payment models that reward value, such as value-based contracts, as well as incentives aligned with quality measures (e.g., CMS star ratings, HEDIS, accountable care organizations). Entities that are at risk for total cost of care also have incentives to integrate data so that they can readily assess total cost of care and implement interventions that influence outcomes.

Transparency of costs, including the effect of rebates, is important for assessment of total costs. Participants noted that revenue streams from rebates are not always transparent and can complicate efforts to implement the most cost-effective interventions. Regulatory strategies may be needed to better align incentives.

Participants observed that there can be competitive advantages to organizations that have integrated data, particularly if they are able to demonstrate the value of their services. Participants also recommended pilot testing programs to assess return on investment for developing integrated datasets and new processes with business partners. Pilots may be used to refine the approach that will work best for a managed care organization to align its priorities, obtain access to necessary data, and update relevant business partner agreements as needed.

\section{Conclusions}

Health care stakeholders identified several potential benefits to enhancing data integration for managing total cost of care, quality of care measures, and population health outcomes. Innovative health care organizations, particularly those that are at risk for total cost of care and quality, have begun to implement systems and practices that facilitate the use of data analytics to better inform care. However, technological, regulatory, financial, and organizational culture barriers hinder more widespread use of such systems. Importantly, participants noted that data integration must be purposeful and provide information that is actionable and useful for informed decision making. 
Through the development of this data integration roadmap, the AMCP forum identified a framework of strategies that organizations can use as they assess their data integration needs and plans. Additionally, forum participants recommended continued stakeholder collaborations and participation with entities that are involved in developing standards for data interoperability. Further, they encouraged managed care organizations to share information about strategies to overcome challenges to data integration, as well as information demonstrating how achieving data integration enables effective and efficient management of the total cost of care.

\section{Forum Participants}

IBRAHIM ABBASS, Research Lead, Genentech; KRISTEN BINASO, Director, Patient Advocacy and Professional Relations, Boehringer Ingelheim Pharmaceuticals; KEVIN BOESEN, CEO, SinfoniaRx; JOEL BRILL, Chief Medical Officer, Predictive Health; JOY BROWN, Vice President, Advanced Analytic Technology Solutions, Optum; AMANDA BRUMMEL, Director, Clinical Ambulatory Pharmacy, Fairview Health Services; JOHN CAI, Executive Director, Real-World Data Analytics and Innovation, Merck; HAE MI CHOE, Chief Quality Officer/Associate Dean, Pharmacy Innovations and Partnerships, University of Michigan Medical Group/University of Michigan College of Pharmacy; SAMMY DOUGHERTY, Deputy Vice President, PhRMA; ERIC ESTES, Senior Director, Chief Pharmacy Officer, Independence Blue Cross; ALYSHA FLUNO, Chief Pharmacy Officer, Truveris; LISA GHOTBI, Director of Pharmacy Services, San Francisco Health Plan; JENNIFER GRAFF, Vice President, Comparative Effectiveness Research, National Pharmaceutical Council; CAROLYN HA, Director, Policy \& Research, PhRMA; MARTHA HOEFER, Manager, Provider Performance, Harvard Pilgrim Health Care; ROB JACOBSON, Executive Director, Value and Access, Amgen; JASON LOTT, Director, Medical Affairs-Market Access, Bayer; KRISTINA LUNNER, Principal, Leavitt Partners; TOM MARTIN, Senior Director, Xcenda; TAMMIE MCGONIGLE, Lead, Medical Specialty Strategy, Aetna; QUYNH-ANH NGUYEN, Manager, Business Intelligence - Pharmacy Analytics, Kaiser Permanente Washington, ANJU PARTHAN, Director, Health Economics and Outcomes Research, Alkermes; BRIAN PELTZ, Managing Principal, Government Programs, Medicaid \& Marketplace, MedImpact Healthcare Systems; VANITA PINDOLIA, Vice President, Ambulatory Clinical Pharmacy Programs, Henry Ford Health System/Health Alliance Plan; ANTHONY SCHUETH, CEO and Managing Partner, Point-of-Care Partners; BONNIE SHAUL, Director, Payer Strategy, AbbVie; CATHERINE STARNER, Health Outcomes Senior Principal Consultant, Prime Therapeutics; DEBBIE STERN, Senior Vice President, Strategy and Business Development, eviCore healthcare; SCOTT STREATOR, Managing Principal, MedImpact Healthcare Systems; ROBIN TURPIN, Value Evidence and Health Outcomes Scientific Lead, Takeda; KEITH WILLARD, Vice President, Chief Architect, Surescripts; and KAT WOLF KHACHATOURIAN, Vice President, Strategic Care Transformation, Qualchoice Health Plan Services.
AMCP Staff: ELISABETH BRISLEY, Legislative Analyst; SUSAN CANTRELL, Chief Executive Officer; MARY JO CARDEN, Vice President, Government and Pharmacy Affairs; ADAEZE CHUKWUKA, APPE Student; CHARLIE DRAGOVICH, Vice President, Strategic Alliance E Corporate Services; JASMINE INMAN, APPE Student; NOREEN MATTHEWS, Consultant; BRIANNA PALOWITCH, Manager, Pharmacy Affairs \& New Practitioner Programs; CYNTHIA REILLY, Senior Vice President; TERRY RICHARDSON, Director, Product Development; RUBY SINGH, Vice President, Education; and TRICIA LEE WILKINS, Director, Pharmacy Affairs.

AUTHOR CORRESPONDENCE: Tricia Lee Wilkins, PharmD, MS, PhD, Academy of Managed Care Pharmacy, 675 N. Washington St., Ste. 220, Alexandria, VA 22314. Tel.: 703.684.2641;

E-mail: tlwilkins@amcp.org.

\section{DISCLOSURES}

The AMCP Partnership Forum and the development of this proceedings document were supported by Abbvie, Alkermes, Amgen, Bayer, Boehringer Ingelheim, Genentech, Merck, National Pharmaceutical Council, PhRMA, Takeda, and Xcenda.

\section{ACKNOWLEDGMENTS}

The stakeholder forum was moderated by Clifford Goodman, Senior Vice President at the Lewin Group. This proceedings document was written by Judy Lofton, Medical Writer and Consultant, JCL Communications. Visual summary was created by Lisa Nelson, CEO and Visual Strategist, See in Colors.

\section{REFERENCES}

1. Advisory Board. Meet the $1 \%$ of patients that account for $23 \%$ of spending. October 10, 2014. Available at: https://www.advisory.com/daily-briefing/2014/10/10/meet-the-1-of-patients-that-account-for-23-of-spending?elq_ cid $=1145532$. Accessed February 13, 2019.

2. HL7 International. Da Vinci Project. Available at: http://www.hl7.org/ about/davinci/index.cfm. Accessed February 13, 2019. 